\title{
Trephine Transverse Colostomy Is Effective for Patients Who Have Previously Undergone Rectal Surgery
}

\author{
Seung-Seop Yeom, Chan Wook Kim, Sung Woo Jung, Se Heon Oh, Jong Lyul Lee, Yong Sik Yoon, In Ja Park, \\ Seok-Byung Lim, Chang Sik Yu, Jin Cheon Kim \\ Departments of Colon and Rectal Surgery, Asan Medical Center, University of Ulsan College of Medicine, Seoul, Korea
}

Purpose: Colostomy creation is an essential procedure for colorectal surgeons, but the preferred method of colostomy varies by surgeon. We compared the outcomes of trephine colostomy creation with open those for the (laparotomy) and laparoscopic methods and evaluated appropriate indications for a trephine colostomy and the advantages of the technique.

Methods: We retrospectively evaluated 263 patients who had undergone colostomy creation by trephine, open and laparoscopic approaches between April 2006 and March 2016. We compared the clinical features and the operative and postoperative outcomes according to the approach used for stoma creation.

Results: One hundred sixty-three patients (62\%) underwent colostomy surgery for obstructive causes and $100(38 \%)$ for fistulous problems. The mean operative time was significantly shorter with the trephine approach (trephine, $46.0 \pm 1.9$ minutes; open, $78.7 \pm 3.9$ minutes; laparoscopic, $63.5 \pm 5.0$ minutes; $\mathrm{P}<0.001)$, as was the time to flatus $(1.8 \pm 0.1$ days, $2.1 \pm 0.1$ days, $2.2 \pm 0.3$ days, $\mathrm{P}=0.025)$. Postoperative complications ( $<30$ days) were not different among the 3 approaches (trephine, $4.3 \%$; open, $1.2 \%$; laparoscopic, $0 \% ; \mathrm{P}=0.828$ ). In patients who underwent rectal surgery, a trephine colostomy was feasible for a diversion colostomy $(\mathrm{P}<0.001)$.

Conclusion: The trephine colostomy is safe and can be implemented quickly in various situations, and compared to other colostomy procedures, the patient's recovery is faster. Previous laparotomy history was not a contraindication for a trephine colostomy, and a trephine transverse colostomy is feasible for patients who have undergone previous rectal surgery.

Keywords: Trephine colostomy; Laparoscopic colostomy; Colostomy; Rectal surgery

\section{INTRODUCTION}

An enterostomy is a purposeful anastomosis between a segment of the gastrointestinal tract and the skin of the anterior abdominal wall [1] that can be created virtually anywhere along the gastrointestinal tract. For diversion of the fecal stream, the most common

Received: April 20, 2017 - Accepted: September 29, 2017

Correspondence to: Chan Wook Kim, M.D.

Department of Colon and Rectal Surgery, Asan Medical Center, University of Ulsan College of Medicine, 88 Olympic-ro 43-gil, Songpa-gu, Seoul 05505, Korea

Tel: +82-2-3010-3928, Fax: +82-2-3010-6701

E-mail: crscwkim@amc.seoul.kr

ORCID code: https://orcid.org/0000-0002-2382-0939

(C) 2018 The Korean Society of Coloproctology

This is an open-access article distributed under the terms of the Creative Commons Attribution NonCommercial License (http://creativecommons.org/licenses/by-nc/4.0) which permits unrestricted noncommercial use, distribution, and reproduction in any medium, provided the original work is properly cited. form of an enterostomy is made using the distal ileum or colon. Temporary or permanent fecal diversions may be needed to manage a variety of pathologic conditions, including congenital anomalies, colon obstruction, inflammatory bowel disease, traumatic disruption of the intestinal tract, gastrointestinal malignancy or metastatic malignancy, as well as complications of rectal surgery [2].

Minimally invasive surgical procedures have been applied with increasing frequency to intestinal operations. Although stoma creation is traditionally achieved via a formal laparotomy, the procedure is well suited as a minimally invasive approach and is now being applied more widely. A minimally invasive approach avoids a large abdominal incision and, therefore, should minimize postoperative pain, ileus, and wound complications. Other advantages may include limited use of postoperative narcotics, shorter hospital stay, and earlier initiation of other therapy, such as chemotherapy or radiation therapy [3-5]. 
Volume 34, Number 2, 2018

In this study, we compared the outcomes of colostomy creation by using the trephine method with those of colostomy creation by using open (laparotomy) and laparoscopic methods. Furthermore, we evaluated the indications for a trephine colostomy and the advantages of the technique.

\section{METHODS}

\section{Patients}

The records of all 482 patients who had undergone a colostomy operation between April 2006 and March 2016 were retrospectively reviewed. All colostomy creations were made by 7 colorectal surgeons with their preferred methods (trephine, open, or laparoscopic). We finally included 263 patients who had undergone a colostomy for the sole purpose of making a diversion colostomy. All pediatric patients and all patients who had undergone a colostomy as a part of a different surgical procedure or had had severe septic conditions were excluded. Patients in whom additional procedures had been executed were also excluded. The protocol for the present study protocol was approved by the Institutional Review Committee of Asan Medical Center (2016-0887) and the informed consent was waived.

The various indications of a colostomy were classified into 2 groups: obstructive or fistulous. Primary colorectal cancer ob- struction, metastatic cancer obstruction, recurred colorectal cancer obstruction, anastomotic stricture, pseudo-obstruction, sigmoid volvulus, and colonic endometriosis were considered obstructive indications. Fistulous indications included rectal fistulae or abscesses due to benign or malignant conditions, delayed anastomotic leakage after rectal surgery, and colonic fistulae.

Patients were divided into 3 groups according to the colostomy procedures: open, trephine, or laparoscopic. The demographics, operation time, length of hospital stay, time to return of bowel function, and early complications were analyzed for each group. The demographics included age, sex, anesthesia method, American Society of Anesthesiologists (ASA) physical status classification grade, and history of previous abdominal surgery.

\section{Operative procedure}

The wound/ostomy, continence nurse evaluated the stoma site prior to surgery. The inguinal area, the umbilicus, the costal margin, skin folds, and scars frequently interfered with stoma care. Leave a 5-cm margin of smooth skin around the stoma is desirable. Potential stoma sites were identified in both lower quadrants and in the left upper quadrant, and the eventual choice was marked with a permanent pen. In all cases, the stoma was placed within the rectus abdominis muscle, which provided support and reduced the incidence of parastomal hernia.
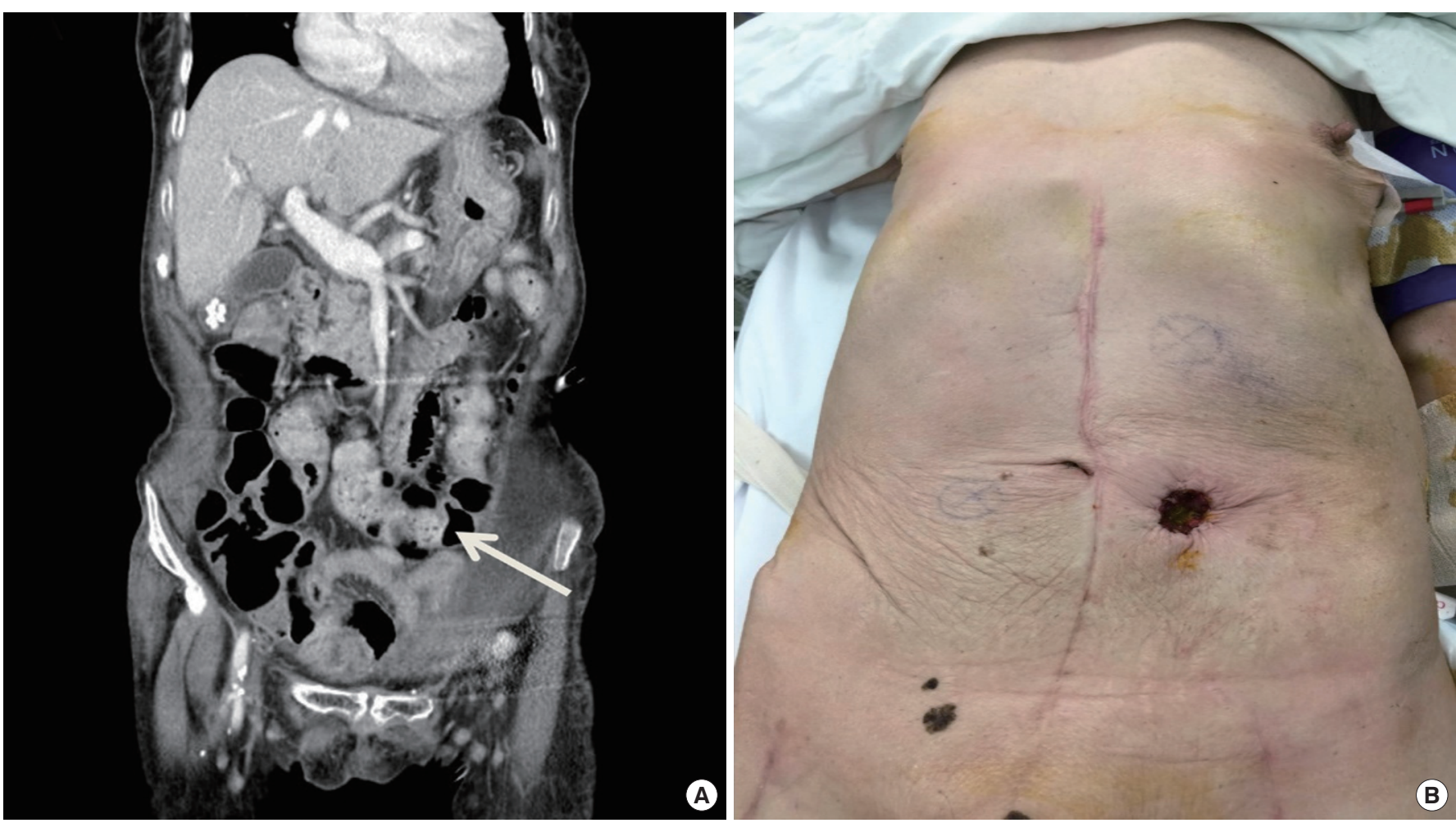

Fig. 1. Case with a redundant transverse colon. (A) The abdominopelvic computed tomography scan demonstrated a redundant transverse colon (arrow). (B) A transverse colostomy was created in the left lower quadrant by using the trephine approach. 


\section{Trephine colostomy}

After careful review of the abdominopelvic computed tomography (CT) images, the abdominal area with a loop of the transverse or sigmoid colon immediately beneath the marked site was identified. In most cases, a transverse colostomy was made in the left upper quadrant. However, in cases with a redundant transverse colon, a transverse colostomy was created in the left lower quadrant (Fig. 1). A disk of skin and subcutaneous tissue was excised to make the opening. This was typically performed within the rectus muscle. A cruciate incision was made in the anterior rectus fascia, the rectus muscle was split along its fibers, and the posterior sheath and peritoneum were incised and opened. If necessary, the opening was extended in the vertical or the horizontal direction. With the use of Babcock's forceps, the transverse or sigmoid colon was grasped and introduced into the wound. In the case of a transverse colon, it was traced along the greater omentum, and the omentum was freed from the colon to allow sufficient exteriorization without tension. In the case of the sigmoid colon, which sometimes had a short mesentery or some peritoneal adhesions, a lateral mobilization with Metzembaum scissors was necessary. The colostomy was anchored to the abdominal wall with sutures, and the seromuscular layer of the colon was fixed to 2 layers of the rectus fascia with interrupted non-absorbable sutures without the use of a stoma rod.

Laparoscopic colostomy

A 12-mm trocar was inserted in the periumbilical area (or colos-

Table 1. Indications for a colostomy in the 263 study patients

\begin{tabular}{lc}
\hline Cause & No. (\%) \\
\hline Obstructive cause & $163(61.9)$ \\
\hline Primary colorectal cancer & $63(24.0)$ \\
\hline Metastatic cancer & $51(19.4)$ \\
Recurred colorectal cancer & $30(11.4)$ \\
Benign cause & $19(7.2)$ \\
Fistulous cause & $100(38.0)$ \\
Perianal disease due to benign disease & $46(17.5)$ \\
Pelvic abscess or fistula after low anterior resection & $42(16.0)$ \\
Rectal fistula due to malignancy & $9(3.4)$ \\
Colonic fistula & $2(0.8)$ \\
Diverticulitis with abscess & $1(0.4)$ \\
\hline
\end{tabular}

Table 2. Characteristics of patients according to type of stoma approach

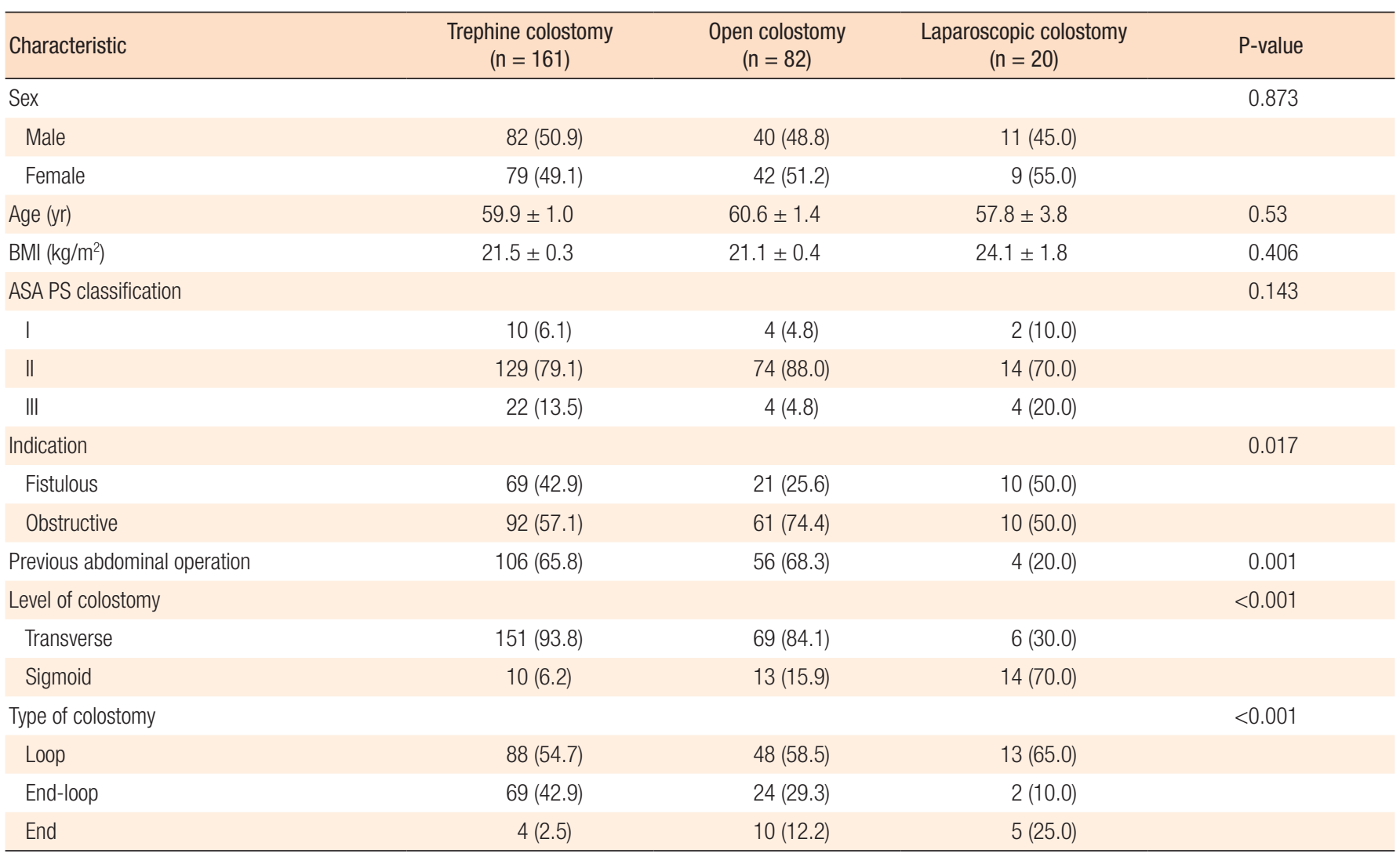

Values are presented as number (\%) or mean \pm standard deviation.

BMI, body mass index; ASA PS, American Society of Anesthesiologists physical status. 
tomy site) to receive the camera. The abdomen was inspected for masses, carcinomatosis, adhesions, and/or any other pathology. Two 5-mm ports were placed in the right upper and lower quadrants of the abdomen to facilitate the identification, dissection, and raising of the limb of the bowel for the stoma.

\section{Maturation of the colostomy}

After the seromuscular fixation to the rectus fascia, stomas were matured with a simple eversion technique using an absorbable suture. This was performed circumferentially and tied down after satisfactory bites had been obtained.

\section{Statistical analysis}

Categorical variables were compared using Fisher exact test or Pearson chi-square test, as appropriate, and continuous variables were compared using the Mann-Whitney U-test or the independent samples t-test. The Kruskal-Wallis test was used for nonparametric analysis of variance. All numbers were expressed as means \pm standard deviations, and $\mathrm{P}<0.05$ was considered statistically significant. Statistical analyses were performed using IBM SPSS Statistics ver. 21.0 (IBM Co., Armonk, NY, USA).

\section{RESULTS}

The indications for a colostomy were obstructive causes in 163 patients (62\%) and fistulous causes in 100 patients (38\%). The causes are detailed in Table 1 . The characteristics of the patients in the 3 treatment groups (open, trephine, and laparoscopic colostomy) are summarized in Table 2. No difference in age, sex, body mass index (BMI), or ASA physical status classification grade was noted among the three groups. The trephine and the open colostomy were more frequently performed for obstructive causes than the laparoscopic colostomy was $(\mathrm{P}=0.017)$. The laparoscopic approach was more frequently performed in patients with no previous history of abdominal surgery $(\mathrm{P}=0.001)$. The trephine colos- tomy was performed mainly to make a transverse colostomy, and a laparoscopic colostomy was performed mainly for a sigmoid colostomy $(\mathrm{P}<0.001)$.

Operative and postoperative outcomes are shown in Table 3. The mean operative time was significantly shorter for the trephine group than for the open and the laparoscopic groups $(46.0 \pm 1.9$ minutes vs. $78.7 \pm 3.9$ minutes vs. $63.5 \pm 5.0$ minutes, $\mathrm{P}<0.001$ ), and the time to flatus was also shortest in the trephine group (1.8

Table 4. Differences in colostomy formation according to history of previous rectal surgery

\begin{tabular}{|c|c|c|c|}
\hline \multirow{2}{*}{ Variable } & \multicolumn{2}{|c|}{ Previous rectal surgery } & \multirow{2}{*}{ P-value } \\
\hline & No $(n=176)$ & Yes $(n=87)$ & \\
\hline Indication & & & $<0.001$ \\
\hline Fistulous & $50(28.4)$ & $50(57.5)$ & \\
\hline Obstructive & $126(71.6)$ & $37(42.5)$ & \\
\hline Level of colostomy & & & $<0.001$ \\
\hline Transverse & 140 (79.5) & $86(98.9)$ & \\
\hline Sigmoid & $36(13.7)$ & $1(1.1)$ & \\
\hline Type of stoma & & & $<0.001$ \\
\hline Loop & 117 (66.5) & $32(36.8)$ & \\
\hline End-loop & $49(27.8)$ & $46(52.9)$ & \\
\hline End & $10(5.7)$ & $9(10.3)$ & \\
\hline Type of approach & & & 0.021 \\
\hline Trephine & $104(59.1)$ & $57(65.5)$ & \\
\hline Open & $53(30.1)$ & 29 (33.3) & \\
\hline Laparoscopic & 19 (10.8) & $1(1.1)$ & \\
\hline Conversion & & & 0.461 \\
\hline Trephine $\rightarrow$ open & $4(2.3)$ & $4(4.6)$ & \\
\hline Trephine $\rightarrow$ laparoscpy & $1(0.4)$ & $0(0)$ & \\
\hline
\end{tabular}

Values are presented as number (\%).

Table 3. Operative and postoperative outcomes according to type of stoma approach

\begin{tabular}{|c|c|c|c|c|}
\hline Variable & $\begin{array}{l}\text { Trephine colostomy } \\
\qquad(\mathrm{n}=161)\end{array}$ & $\begin{array}{l}\text { Open colostomy } \\
\quad(\mathrm{n}=82)\end{array}$ & $\begin{array}{l}\text { Laparoscopic colostomy } \\
\qquad(\mathrm{n}=20)\end{array}$ & P-value \\
\hline Operation time (min) & $46.0 \pm 1.9$ & $78.7 \pm 3.9$ & $63.5 \pm 5.0$ & $<0.001$ \\
\hline Hospital stay (day) & $11.4 \pm 1.4$ & $9.8 \pm 0.8$ & $8.9 \pm 1.0$ & 0.474 \\
\hline Time to flatus (day) & $1.8 \pm 0.1$ & $2.1 \pm 0.1$ & $2.2 \pm 0.3$ & 0.025 \\
\hline Complication & & & & 0.828 \\
\hline Prolapse & $4(2.5)$ & $1(1.2)$ & $0(0)$ & \\
\hline Retraction & $2(1.2)$ & $0(0)$ & $0(0)$ & \\
\hline Kinking & $1(0.6)$ & $0(0)$ & $0(0)$ & \\
\hline \multicolumn{5}{|l|}{ Conversion } \\
\hline Open & $7(4.3)$ & - & $1(5)$ & \\
\hline Laparoscopic & - & - & - & \\
\hline
\end{tabular}

Values are presented as mean \pm standard deviation or number (\%). 
\pm 0.1 days vs. $2.1 \pm 0.1$ days vs. $2.2 \pm 0.3$ days, $\mathrm{P}=0.025)$. No differences in the length of hospital stay or the incidence of complications were found among the 3 groups. During the trephine procedure, 7 of 161 cases $(4.3 \%)$ were converted to a laparotomy. During the laparoscopic operation, 1 of 20 cases (5\%) was converted to a laparotomy.

Of the 263 patients, 87 (33.0\%) had a previous history of rectal surgery. Among these 87 patients, most causes for the colostomy were fistulous problems $(\mathrm{P}<0.001)$ (Table 4$)$, and transverse loop or end-loop colostomies were more often used $(\mathrm{P}<0.001)$. The trephine colostomy was the most preferred method for 87 patients with a history of surgery, and laparoscopic approaches were rarely used.

\section{DISCUSSION}

The trephine and the laparoscopic stoma techniques can be applied for an ileostomy or a colostomy of an end or a loop type. In this study, trephine and laparoscopic colostomies were found to be successful in 173 of 181 cases (95.6\%). Seven of the trephine colostomies (4.3\%) were converted to open surgery. The reasons for conversion were adhesions in five cases and bowel dilatation with a thick abdominal wall in 2 cases. For 1 patient undergoing a laparoscopic colostomy, the procedure was switched to open surgery due to bowel dilatation and a thick abdominal wall.

In previous studies, adhesion was the main reason for conversion to open surgery [3, 6-9]. Among studies about the trephine colostomy, the rates of conversion were reported to be $6 \%-29 \%$ $[3,5,9,10]$. In this study, 7 cases $(4.3 \%)$ were converted to a laparotomy during a trephine colostomy, a relatively lower rate than previously reported. Especially in the trephine colostomy group, among 106 patients who had a history of abdominal surgeries, the trephine colostomy was feasible for 101 patients (95.3\%).

Two cases were converted from a trephine colostomy to a laparotomy because of obesity. One case resulted from a thick abdominal wall and severe bowel dilatation; the patient's BMI was 33.36 $\mathrm{kg} / \mathrm{m}^{2}$. The other case resulted from a pseudo-obstruction of colon; in this case, the patient's with a BMI of $34.28 \mathrm{~kg} / \mathrm{m}^{2}$. In a previous study, CT-guided stoma formation was reported to be beneficial [11]; similarly, we did a careful review of the abdominopelvic $\mathrm{CT}$ before a trephine colostomy to identify the target colon. This was very useful for determining the appropriate colostomy method.

In this study, the mean operation times and the recovery times were significantly shorter for patients who underwent a trephine colostomy; however, the lengths of hospital stay were not statistically different among the 3 colostomy methods. Although many previous studies included hospital stay as a variable for comparing colostomy methods, this variable is really not a meaningful one because hospital stay is determined by the original disease, which is reason for the colostomy, not by the colostomy. For example, in patients with perineal infection, long hospital stays are required for infection control, even after the stoma function. Thus, we believe that bowel function recovery time is a more reliable variable for comparing colostomy modalities.

The trephine colostomy has been reported to have many benefits, including limited abdominal incisions and the ability to be performed under local or regional anesthesia $[3,12]$. However, it does have disadvantages: the small incision makes orienting the bowel difficult and inspecting the abdominal cavity impossible. Furthermore, potential pitfalls of the technique include retraction of the stoma, difficulty in identifying the proximal loop, prolapse, and stricture $[10,13,14]$. One study reported complication rates as high as $25 \%$ [15] while in the current study, only 7 complications $(4.3 \%)$ occurred after a trephine colostomy, and the difference between the open and the laparoscopy approaches was not significant $(\mathrm{P}=0.828)$. The stoma-related complications were known to have occurred after the period of physiologic adjustment. For most patients, complications occur between 6 and 10 weeks after the surgery. One large series found that $81 \%$ (448 of 553 ) of the complications occurred within the first month postoperatively [16]. Another series identified that the actuarial risks of parastomal complications were $58.1 \%$ until 13 years postoperatively [17].

In our institution, 7 surgeons used different methods for a colostomy, open, trephine, and/or laparoscopic. In our experience, the surgeons who prefer a trephine colostomy, rarely encountered complications, and their patients had fast recovery after surgery; this was the motivation driving this study. At our institution, before surgery, our surgeons review abdominal CT images very carefully; if severe adhesions are suspected or the target colon is thought to be too short to lift up, the surgeons remove the trephine method from consideration. Nevertheless, we had no hard data to support drawing any conclusions based on our experience, so we tried to evaluate our colostomy methods and to find indications for their use. The present study was designed to compare the short-term operative outcomes ( $<30$ days) and the recoveries of the patients; thus, accurate interpretations of the complication rates were limited. If the complications rates are to be interpreted accurately, long-term follow-up studies will be necessary.

In this study, the trephine and the open methods mainly used the transverse colon for diversion colostomies while laparoscopic colostomy creation mainly used the sigmoid colon. This was most likely related to the previous history of surgery. Of the patients who had undergone surgery in the past, $52.4 \%$ (87 of 166) had a history of the rectal surgery, and patients who have undergone rectal surgery will usually have a relatively shorter sigmoid colon, thus rendering colostomy creation at this site difficult. In those cases, the transverse colon was a more feasible site for a diversion colostomy and had the further advantage of the surgeon's being able to determine the direction of colon through verification of the direction of the omental attachment.

This study has many limitations. First, inclusion criteria were not strictly controlled because our data was not collected prospec- 
tively. Nevertheless, the facts that this was the first study comparing three different methods of colostomy creation, that it was one of the largest comparative study to date, and that data were analyzed several ways make this a meaningful study. Second, although trephine stoma creation showed superior operation and recovery times, the open and the laparoscopic method still had substantial benefits of abdominal exploration.

This study showed that all three colostomy methods could be safely performed with low complication rates. Especially, the trephine colostomy was implemented safely and quickly in various situations and its use was feasible for patients who had undergone previous open abdominal surgeries. Furthermore, the trephine transverse colostomy was a useful method for patients who had previously undergone rectal surgery. Nevertheless, well-designed studies on the delicate indications for and the long-term outcomes of a trephine colostomy are needed.

\section{CONFLICT OF INTEREST}

No potential conflict of interest relevant to this article was reported.

\section{REFERENCES}

1. Cataldo PA. Technical tips for stoma creation in the challenging patient. Clin Colon Rectal Surg 2008;21:17-22.

2. Doughty D. Principles of ostomy management in the oncology patient. J Support Oncol 2005;3:59-69.

3. Stephenson ER Jr, Ilahi O, Koltun WA. Stoma creation through the stoma site: a rapid, safe technique. Dis Colon Rectum 1997;40: 112-5.

4. Young CJ, Eyers AA, Solomon MJ. Defunctioning of the anorec- tum: historical controlled study of laparoscopic vs. open procedures. Dis Colon Rectum 1998;41:190-4.

5. Anderson ID, Hill J, Vohra R, Schofield PF, Kiff ES. An improved means of faecal diversion: the trephine stoma. Br J Surg 1992;79: 1080-1.

6. Hollyoak MA, Lumley J, Stitz RW. Laparoscopic stoma formation for faecal diversion. Br J Surg 1998;85:226-8.

7. Ludwig KA, Milsom JW, Garcia-Ruiz A, Fazio VW. Laparoscopic techniques for fecal diversion. Dis Colon Rectum 1996;39:285-8.

8. Hellinger MD, Al Haddad A. Minimally invasive stomas. Clin Colon Rectal Surg 2008;21:53-61.

9. Nylund G, Oresland T, Hultén L. The trephine stoma: formation of a stoma without laparotomy. Eur J Surg 1997;163:627-9.

10. Patel P, Wright A, Messersmith R, Palmer J. Does trephine colostomy produce a satisfactory stoma? Colorectal Dis 2001;3:270-1.

11. Craig E, Garner J, Amin S. Stoma siting using CT guidance in a multiply scarred abdomen. Tech Coloproctol 2007;11:281-2.

12. Soyder A, Özgün H. Trephine ostomy: safe and simple. Surgery Curr Res 2013;3:1000139.

13. Jess P, Christiansen J. Laparoscopic loop ileostomy for fecal diversion. Dis Colon Rectum 1994;37:721-2.

14. Senapati A, Phillips RK. The trephine colostomy: a permanent left iliac fossa end colostomy without recourse to laparotomy. Ann R Coll Surg Engl 1991;73:305-6.

15. Kini SU, Perston Y, Radcliffe AG. Laparoscopically assisted trephine stoma formation. Surg Laparosc Endosc 1996;6:371-4.

16. Park JJ, Del Pino A, Orsay CP, Nelson RL, Pearl RK, Cintron JR, et al. Stoma complications: the Cook County Hospital experience. Dis Colon Rectum 1999;42:1575-80.

17. Londono-Schimmer EE, Leong AP, Phillips RK. Life table analysis of stomal complications following colostomy. Dis Colon Rectum 1994;37:916-20. 